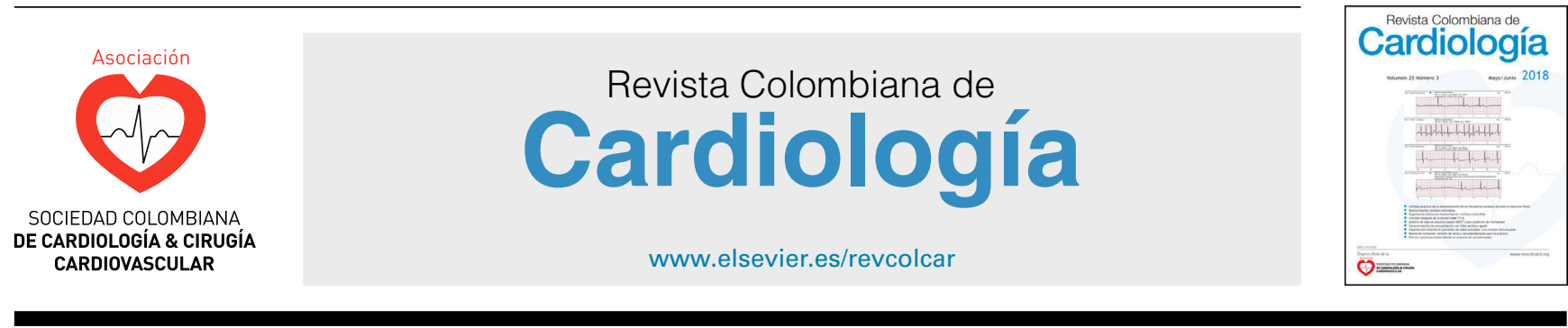

\title{
EDITORIAL
}

\section{"Errar es humano. Ocultar los errores es imperdonable. No aprender de ellos, no tiene justificación"}

\author{
"To err is human. To cover up is unforgivable. To fail to learn is \\ inexcusable."
}

\section{Darío Echeverri}

\author{
Revista Colombiana de Cardiología
}

Recibido el 4 de octubre de 2018; aceptado el 5 de octubre de 2018

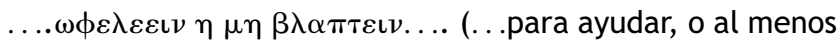
no hacer daño...)

Hipócrates, Epidemics, Bk. I, Sect. XI, c. 400 B.C.

Es muy frecuente que cuando nos encontramos en frente de nuestros pacientes, colegas, alumnos o simplemente cuando estamos recopilando resultados de nuestras observaciones para escribir un artículo científico, al momento de tener en cuenta los efectos adversos o las complicaciones que se presentan sintamos preocupación y se generen diferentes sentimientos de frustración.

A la luz de la práctica médica actual, y en especial de la Cardiología y la Cirugía Cardiovascular, foco temático de esta Revista Colombiana de Cardiología, cada día nos enfrentamos a pacientes más complejos, prematuros o ancianos, con multi-morbilidades (diabetes, enfermedades mentales, pulmonares, renales, hepáticas, fragilidad, etc.), polifarmacia (diuréticos, vasodilatadores, anticoagulantes, inhibidores de bomba de protones, antiarrítmicos, analgésicos, etc.), re-intervenidos varias veces y sometidos a intervenciones percutáneas diversas (marcapasos, desfibriladores, stents, válvulas percutáneas, implante de MitraClips, cierre de apéndice auricular izquierdo,

Correo electrónico: decheverri@cardioinfantil.org endoprótesis de aorta, cierres percutáneos de defectos intracardiacos, etc.), cirugías de revascularización miocárdica, y muchos más, y el uso de nuevos medicamentos, dietas, productos homeopáticos que salen al mercado para el tratamiento y control de condiciones especiales, incrementando la posibilidad de eventos adversos y iatrogenia, que además ponen en riesgo la salud del paciente y su la vida, aumentando los tiempos de hospitalización y los reingresos y, finalmente, los costos al sistema de salud.

Son abundantes los artículos que hablan de la eficacia de todas estas terapias, pero muy pocos los que describen profundamente la seguridad. Los denominados eventos adversos son aquellos que ocurren durante el cuidado clínico y resultan de daño físico o psicológico secundario a iatrogenia, y no del curso natural de la enfermedad o de condiciones propias del paciente. La iatrogenia ha sido parte de la medicina desde sus inicios y continuará haciendo parte de ella mientras exista como arte y ciencia.

Enormes avances se han hecho en la medicina. Sin embargo, solo fue hasta la década de los años 60, cuando se describieron los efectos de la talidomida y los estrógenos sintéticos (diethylestilbestrol) y la Administración de Alimentos y Medicamentos (FDA), en los Estados Unidos, alertó y describió los efectos adversos de estos dos medicamentos. El desarrollo de la epidemiología y la elaboracion de estudios 
clínicos basados en el método cientifico, la aleatorización y la utilizacion de grupos control han ayudado a identificar la verdadera eficacia y seguridad de múltiples terapias.

El Journal of Patient Safety, informó el verdadero número de muertes prematuras asociadas con daños prevenibles, estimado en más de 400.000 por año. El daño grave parece ser de diez a veinte veces más común que el daño letal. Según los hallazgos presentados en el informe del año 2014 por parte de National Partnership for Patients (P4P), de 11 áreas tenidas en cuenta, 5 redujeron los riesgos. Esto representó una reducción de 15.500 muertes y el ahorro de 3.1 a 4 billones de dolares en comparación con el informe de 2010.

Hoy en día, el concepto de iatrogenia ha venido ganando interés y popularidad en la investigación clínica. Si bien desde los inicios de la medicina la iatrogenia ha sido una causa "natural" de enfermedad, en la actualidad la sociedad no está contenta con la situación y razonablemente exige el cumplimiento de una nueva cultura que le ofrezca la sensación de "paciente seguro" como un compromiso de todos los miembros del cuidado integral en salud. El uso de scores de riesgo, la identificación de pacientes complejos, la creación de grupos de atención integral y el análisis de las causas de los errores médicos deben llevar a procesos de mejoramiento.

A pesar de la explosión de la informacion científica, vivimos muchas condiciones adversas relacionadas con medicamentos y dispositivos que permanecen en la vida real y ponen aun más en riesgo a nuestros pacientes: la mayoría de medicamentos actúan mediante un mecanismo de aumento o disminución de dosis en lugar de intentar normalizar la patología para lo cual fueron creados, existe una mínima o nula consideración de los mecanismos compensatorios innatos del cuerpo por parte del médico, no se comprenden por completo los efectos de la polifarmacia, especialmente con opiáceos, analgésicos, pastillas para dormir, antidepresivos y anticoagulantes orales; en general, los pacientes tienen baja adherencia a los medicamentos y a los consejos para tener un de estilo de vida adecuado; los suplementos dietéticos sin receta, los productos a base de hierbas y las terapias alternativas no tradicionales pueden interferir con los medicamentos recetados; pueden darse interacciones entre estos medicamentos, y finalmente, la publicidad indiscriminada dirigida al consumidor, especialmente en radio y televisión, alienta a los pacientes a pedir medicamentos específicos por su nombre, creando así un diálogo directo entre el consumidor y la compañía farmacéutica, separando al médico de la "ecuación".

Estos pocos renglones, son solamente un llamado de atención a los médicos, investigadores y docentes para tener en cuenta la seguridad del paciente, hacer todo lo posible para reducir la posibilidad de eventos adversos, incluir en los programas de formación los temas de iatrogenia y cardiotoxicidad, insistir en las indicaciones adecuadas para uso de medicamentos y realización de procedimientos invasivos e implante indiscriminado de dispositivos, hacer un seguimiento clínico exhaustivo, recopilar la información y publicarla para el bien de nuestros pacientes y para la protección del sistema de salud.

\section{Bibliografía recomendada}

1. Gussak ML, Gussak IB, Kostis JB. Iatrogenicity. Definition, history, and modern context. En: Gussak IB, Kostis JB, editores. latrogenicity causes and consecuences of iatrogenesis in cardiovascular medicine. USA: Rutgers; 2018. p. 5-15.

2. James JT, new A. evidence-based estimate of patient harms associated with hospital care. J Patient Saf. 2013;9:122-8.

3. Project Evaluation Activity in Support of Partnership for patients: Task 2 Evaluation Progress Report. February 2014. Disponible en: https://innovation.cms.gov/Files/reports/PFPEvalProgRpt.pdf.

4. Wilson JW. Unintended consequences avoiding restenosis and stent thrombosis. Texas Heart Institute Journal. 2010;37:341-2. 